\title{
Pterobothrium crassicolle (Eucestoda: Trypanorhyncha) em corvinas, Micropogonias furnieri, comercializadas no município de Niterói, Rio de Janeiro, Brasil
}

\section{Pterobothrium crassicolle (Eucestoda: Trypanorhyncha) in croacker, Micropogonias furnieri, purchased at Niterói municipality, Rio de Janeiro, Brazil}

\author{
Carlos José da Silva Porto, ${ }^{*}$ Sérgio Carmona de São Clemente, ${ }^{* *}$ Mônica Queiroz de Freitas, ${ }^{* *}$ \\ Ricardo Raphael Bastos de São Clemente, ${ }^{\star \star \star}$ Marcelo Knoff, ${ }^{* \star *}$ Edilson Matos ${ }^{\star \star \star \star *}$
}

\begin{abstract}
Resumo
Micropogonias furnieri (Desmarest, 1823) é uma das espécies de peixes mais consumidas pela população do estado do Rio de Janeiro. Entre os meses de maio e setembro de 2006, 30 espécimes deste pescado foram obtidos do mercado de São Pedro no município de Niterói, estado do Rio de Janeiro. Eles foram necropsiados e analisados para presença de cestóides Trypanorhyncha. Um total de oito espécimes apresentou no mesentério plerocercos de Pterobothrium crassicolle, com prevalência de $26,7 \%$, intensidade média de infecção de 2,25 , variando de uma a três larvas por peixe e a abundância média de 0,6 . Os resultados obtidos no presente trabalho são analisados e comparados com os registros anteriores estudados sobre esta espécie de peixe no Brasil.
\end{abstract}

Palavras-chave: Pterobothrium crassicolle, Micropogonias furnieri, corvina.

\begin{abstract}
Porto,C.J.S., São Clemente, S.C., Freitas, M.Q., São Clemente R.R.B., Knoff. M. and Matos, E. [Pterobothrium crassicolle (Eucestoda: Trypanorhyncha) in croacker, Micropogonias furnieri, purchased at Niterói municipality, Rio de Janeiro, Brazil.] Micropogonias furnieri (Desmarest, 1823) is one of the species of fish most consumed by the population of the State of Rio de Janeiro. Between May and September 2006, 30 specimens of this fish were obtained from the market of San Pedro in Niterói municipality, State of Rio de Janeiro. They were necropsied and examined for the presence of cestodes Trypanorhyncha. A total of eight specimens showed the mesentery with plerocerci of Pterobothrium crassicolle, with a prevalence of $26.7 \%$, mean intensity of infection of 2.25, ranging from one to three larvae per fish and mean abundance of 0.6 . The results obtained in this study are analyzed and compared with previous records on the studied fish species in Brazil.
\end{abstract}

Keywords: Pterobothrium crassicolle, Micropogonias furnieri, croacker.

\section{Introdução}

A corvina, Micropogonias furnieri (Desmarest, 1823), é uma espécie costeira encontrada em fundos lodosos e arenosos, mais comumente em profundidades inferiores a 60 metros, e se alimentam de organismos planctônicos nas primeiras fases de desenvolvimento; e essa espécie apresenta um aspecto alimentar bastante diversificado, o qual é constituído por elementos componentes das comunidades demersais e bentônicas nas demais fases (Menezes e Figueiredo, 1980). Esta espécie de peixe possui valor comercial significativo e é uma das mais consumidas pela população do estado do Rio de Janeiro. Sob o ponto de vista da saúde pública, os helmintos da ordem Trypanorhyncha causam repugnância ao consumidor pela sua presença nos filés e cavidade geral dos peixes, levando-os ao descarte, e consequentemente determinando prejuízos econômicos. A espécie Pterobothrium crassicolle tem sido registrada no Brasil de alguns hospedeiros teleósteos de água doce e marinhos, como componente da comunidade parasitária de M. furnieri coletados do litoral do estado do Rio de Janeiro e Rio Grande do Sul (Pereira Jr e Boeger, 2005). O objetivo do presente trabalho foi analisar a presença de cestóides da ordem Trypanorhyncha e determinar as espécies e seus índices parasitários, em corvinas, M. furnieri, comercializadas no município de Niterói, no estado do Rio de Janeiro.

\footnotetext{
Faculdade de Biologia Marinha, Faculdades Integradas Maria Thereza - FAMATH.

** Faculdade de Veterinária, Universidade Federal Fluminense, Rua Vital Brazil, 64, Vital Brazil, CEP 24230-340, Niterói, RJ, Brasil. e-mail: scsc@vm.uff.br

*** Universidade do Rio de Janeiro, UNIRIO.

**** Laboratório de Helmintos Parasitos de Vertebrados, Instituto Oswaldo Cruz, FIOCRUZ.

*****Universidade Federal Rural da Amazônia - UFRA.
} 


\section{Material e métodos}

Entre maio e setembro de 2006 foram coletadas 30 espécimes de corvina (20,5-30,0 de comprimento total), $M$. furnieri, adquiridos no mercado de São Pedro no município de Niterói, estado do Rio de Janeiro. Os peixes foram embalados em sacos plásticos individualmente, acondicionados em recipientes térmicos a baixas temperaturas e transportados para o laboratório onde foram descamados e necropsiados para a inspeção da cavidade abdominal e dos filés, seguindo-se da coleta dos blastocistos, os quais foram rompidos com auxílio de estiletes ao estereomicroscópio. Posteriormente, os cestóides foram colocados em placas de Petri contendo água destilada e transferidos para a refrigeração por um período de 24 horas a fim de morrerem com os tentáculos distendidos, fixados em A.F.A., corados em carmin alcoólico de Langeron, diferenciados em creosoto de Faia e montados em bálsamo do Canadá. Os procedimentos de necropsia e preparação dos helmintos foram realizados segundo Amato et al. (1991). A classificação taxônomica dos cestóides foi baseada em Campbell e Beveridge (1994) e a determinação específica em São Clemente (1986a), Rego (1987) e Campbell e Beveridge (1996). Os índices parasitários de prevalência e intensidade média de infecção foram calculados segundo Bush et al. (1997).

\section{Resultados}

Dos 30 peixes coletados 8 apresentaram plerocercos de cestóides Trypanorhyncha no mesentério, pela espécie Pterobothrium crassicolle (Figuras 1 e 2). A sua prevalência foi de $26,7 \%$, a intensidade média de infecção de 2,25, variando de um a três larvas por peixe e a abundância média de 0,6 . Não foi identificada nenhuma outra espécie de Trypanorhyncha. O tamanho e formato do blastocisto conferem um aspecto repugnante ao consumidor.

\section{Discussão}

Plerocercos de $P$. crassicolle têm sido registrados em nossos teleósteos, inclusive em corvinas, em várias regiões do Brasil (Pereira Jr e Boeger, 2005). Assim os índices parasitários de Micropogonias furnieri e sítios de infecção apresentados no presente trabalho, que ocorreram em infecções simples, puderam ser confrontados com os registros existentes de algumas destas regiões. São Clemente (1986a, b) realizou coletas em 1000 peixes provenientes do município do Rio de Janeiro, estado do Rio de Janeiro, onde esta espécie de cestóide apresentou prevalência de $11 \%$, intensidade média de 1,52 e abundância média de 0,17 (índices pouco abaixo dos registrados no presente trabalho, mas muito próximos), mas que se apresentou coocorrente com outras três espécies de Trypanorhyncha, Pterobothrium heteracanthum, Poecilancistrium caryophyllum e Callitetrarhynchus gracilis, diferentemente do apresentado no presente estudo. No litoral do estado do Rio Grande do Sul estes registros se deram por duas vezes e as amostras foram provenientes de duas localidades, em Pereira Jr. (1993) foi realizada a coleta de 179 peixes no estuário da Lagoa dos Patos onde apresentou prevalência de $10 \%$ e intensidade média de 3,33 (índices muito próximos aos registrados no presente trabalho). Entretanto, apresentou-se coocorrente com outras quatro espécies de Trypanorhyncha, Pterobothrium heteracanthum, Poecilancistrium caryophyllum, Callitetrarhynchus gracilis e C. speciosus, diferente do apresentado no presente trabalho; e em Pereira Jr. e Boeger (2005) coletaram 180 peixes no município de Rio Grande, com prevalência de 66,7 \% e intensidade média de 5,2 e abundância média de 0,17 (prevalência bem mais elevada do que foi observada no presente trabalho, porém intensidade média e abundância média similarmente baixas a que já vinham sendo registradas e com o presente trabalho) e coocorrente com outras cinco espécies de Trypanorhyncha, Dollfusiella sp.,

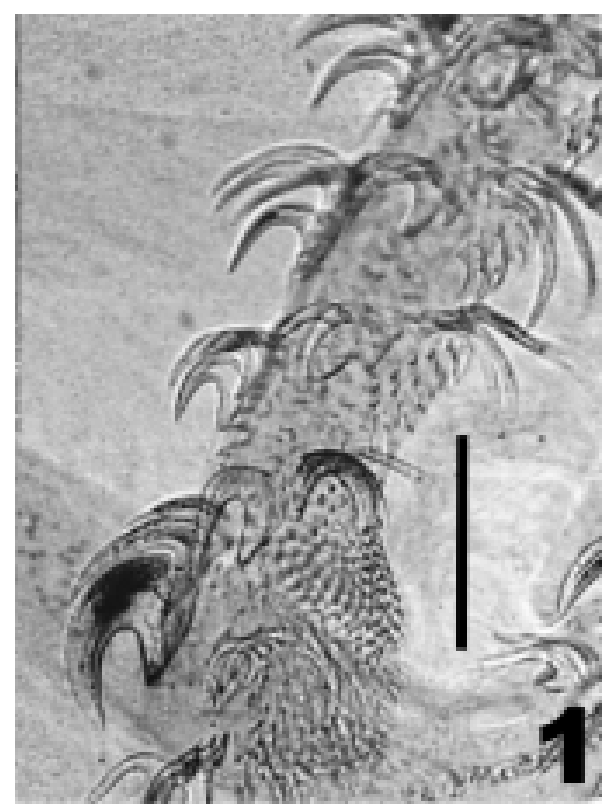

Figura 1:Escólice de $P$. crassicolle oncotaxia da região basal externa. Barra das figuras $=100 \mu \mathrm{m}$.

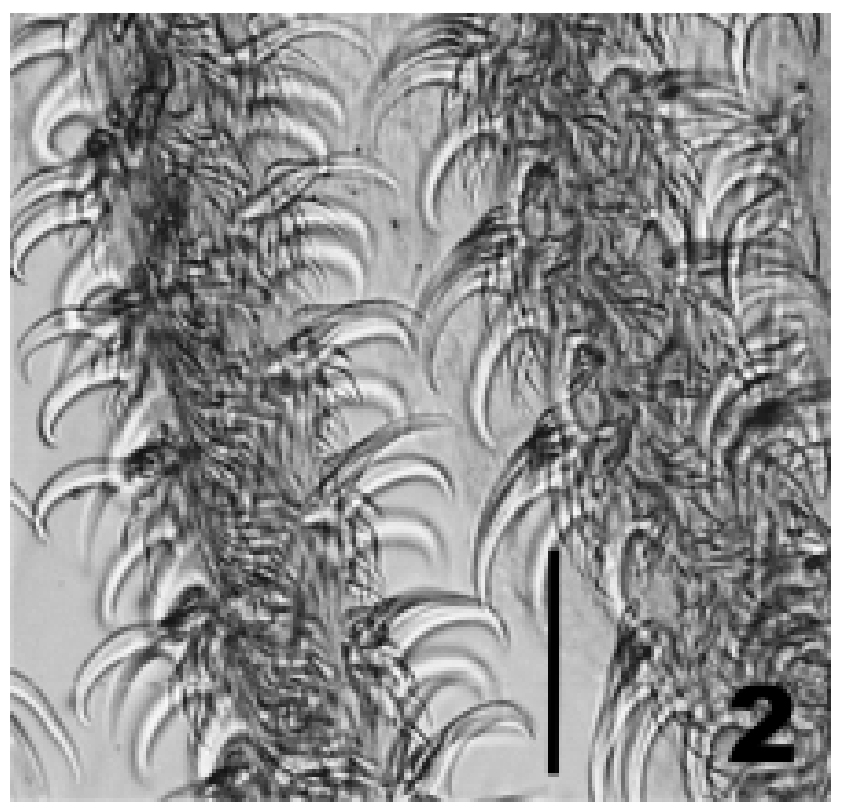

Figura 2: Escólice de P.crassicolle, oncotaxia da região meta basal externa. Barra das figuras $=100 \mathrm{im}$. 
Progrillotia dollfusi, Pterobothrium heteracanthum, Poecilancistrium caryophyllum, Callitetrarhynchus gracilis e C. speciosus, diferente do apresentado no presente trabalho. Interessante notar que no presente estudo este plerocerco de Trypanorhyncha foi coletado apenas em infecções simples sem a presença de nenhum outro cestóide. Entretanto, o número bem menor de coletas pode ter sido o fator determinante influenciando nesta análise. A confirmação da ocorrência desta espécie de Trypanorhyncha em corvinas $M$. furnieri coletadas em mais um município do estado do Rio

\section{Referências}

AMATO, J.F.R.; BOEGER, W.A.; AMATO, S.B. Protocolos para laboratório - coleta e processamento de parasitos de pescado. Seropédica: Imprensa Universitária, Universidade Federal Rural do Rio Janeiro, Rio Janeiro, 1991. 81 p.

BUSH, A.O; LAFFERTY, K.D.; LOTZ, J.M. ; SHOSTAK, A.W. Parasitology meets ecology on its own terms: Margolis et al. Revisited. J Parasitol, v. 83, n. 4, p. 575-583, 1997.

CAMPBELL, R.A.; BEVERIGDE, I. Order Trypanorhyncha. In: Khalil, L.F.; Jones, A.; Bray, R.A. (Eds.). Keys to the Cestode Parasites of Vertebrates. St. Albans: CAB International, 1994, p. 51-148.

CAMPBELL, R.A.; BEVERIDGE, I. Revision of the family Pterobothriidae Pintner, 1931 (Cestoda: Trypanorhyncha). Invertebr Taxon, v. 10, n. 1, p. 617-662, 1996.

MENEZES, N.A. ; FIGUEIREDO, J.L. Manual de peixes marinhos do sudeste do Brasil. IV Teleostei (3). Museu de Zoologia, Universidade de São Paulo, São Paulo, 1980. 96 p.

PEREIRA JR, J. O complexo de espécies de Trypanorhyncha (Cestoda), em corvinas Micropogonias furnieri do Rio Grande do Sul. Arq Fac Vet UFRGS, v. 21, p. 58-70, 1993. de Janeiro enfatiza a atuação deste peixe como importante hospedeiro intermediário no nosso país. Estes cestóides não causam zoonose, mas a presença deste plerocerco nessa espécie de peixe pode levá-lo ao descarte pela vigilância sanitária em decorrência do aspecto repugnante que apresenta. Por isto, é recomendado que se realize a evisceração do pescado antes de serem levados a comercialização, ressaltando a importância na inspeção sanitária deste pescado, como já havia sido salientado por São Clemente (1987).

PEREIRA JR, J.; BOEGER, W.A. Larval tapeworms (Platyhelminthes, Cestoda) from sciaenid fishes of the southern coast of Brazil. Zoosystema, v. 27, n. 1, p. 5-25, 2005.

REGO, A.A.; SANTOS, J.C. ; SILVA, P.P. Estudos de cestóides de peixes do Brasil 1. Mem Inst Oswaldo Cruz, v. 72, n. 3-4, p. 187-204,1974.

REGO, A.A. Redescrição de Pterobothrium crassicolle Diesing, 1850 (Cestoda: Trypanorhyncha) e revalidação de espécie. Mem Inst Oswaldo Cruz, v. 82, n. 1, p. 51-53, 1987.

SÃO CLEMENTE, S.C. Plerocercos da ordem Trypanorhyncha parasitos de corvina Micropogonias furnieri (Desmarest) no litoral do Estado do Rio de Janeiro. Atas Soc Biol Rio de Janeiro, v. 26, p. 29-36, 1986a.

SÃO CLEMENTE, S.C. Prevalência e intensidade média de infecção de plerocercos de Trypanorhyncha parasitando Corvina Micropogonias furnieri (Desmarest) no litoral do Estado do Rio de Janeiro. Atas Soc Biol Rio de Janeiro, v. 26, p. 37-40, 1986b.

SÃO CLEMENTE, S.C. Plerocercos de cestóides da ordem Trypanorhyncha em corvina Micropogonias furnieri e sua importância na inspeção sanitária do pescado. Arq Fluminenses Med Vet, v. 2, p. 82-83, 1987. 